\title{
Surface Phonons Modes: A Tool to Determine the Quantum Dot Morphology
}

\author{
Augusto M. Alcalde, Alessandra A. Ribeiro, Noélio O. Dantas, \\ Instituto de Física, Universidade Federal de Uberlândia, 38400-902, Uberlândia, MG, Brazil \\ Djalma R. Mendes Jr., \\ Instituto de Física, Universidade de São Paulo, 05315-970, São Paulo, SP, Brazil \\ Gilmar E. Marques, \\ Departamento de Física, Universidade Federal de São Carlos, 13565-905, São Carlos, SP, Brazil \\ and Carlos Trallero-Giner \\ Departamento de Física Teórica, Universidad de la Habana, Vedado 104000, Havana, Cuba
}

Received on 8 December, 2005

\begin{abstract}
We report here theoretical and experimental studies on the spatial confinement of phonons in ternary $\mathrm{CdS}_{x} \mathrm{Se}_{1-x}$ nanocrystals embedded in a glass matrix formed by the composites $\mathrm{SiO}_{2}-\mathrm{Na}_{2} \mathrm{CO}_{3}-\mathrm{B}_{2} \mathrm{O}_{3}-\mathrm{Al}_{2} \mathrm{O}_{3}$ doped with $\mathrm{CdO}, \mathrm{S}$ and $\mathrm{Se}$. We determined the morphologic characteristics of the nanocrystals by analyzing the dependence of surface phonon modes on the geometrical parameters. The calculated frequencies are compared with values from Raman spectra of $\mathrm{CdS}_{x} \mathrm{Se}_{1-x}$ nanocrystals grown under different thermal treatments. A good correlation between experimental and calculated CdS-like and CdSe-like surface optical modes is observed. Raman selection rules and their connection with the nature of the surface optical phonons is discussed.
\end{abstract}

Keywords: Quantum dots; Raman scattering

The quantum confinement effects on the optical properties of nanocrystallites or quantum dots (QD's) grown over semiconductor-doped glasses are subject of current researches. The interest in these materials arises from their great potential for use in device applications due to their nonlinear optical properties. Experimental and theoretical studies showing the confinement effects on the QD electronic states and their energy levels are now well established. Nonetheless, the size effects on their phonon modes and on the electron-phonon interaction are still somewhat controversial. In this theoretical scenario, one aspect has not yet been sufficiently addressed: how the geometrical shape effects can modify the electronic structure and other physical properties in small QD's. In IIVI QD's, the morphological effects can modify the energies, symmetry properties of the wave functions and, thus, their optical transitions.

In this work, we explore theoretically and experimentally the connection between the surface optical (SO) phonon modes and the geometrical shape of a semiconductor QD. By analyzing the behavior of SO modes in ternary alloy based QD's, we may determine the alloy composition fluctuation and the geometrical parameter $r$, that measures the degree of deviation from sphericity. To do this, we have extended the Comas et al. [1] model for binary spheroidal dots to study ternary alloys of $A_{1-x}^{1} A_{x}^{2} B$ QD type. Thus, we calculated the dependence of the $A^{1} B$-like and $A^{2} B$-like SO-modes as a function of $x$ and $r$. Our extended theory recovers the binary compound limits $x \rightarrow 0$ and $x \rightarrow 1$ of Ref. [1] and verifies the conditions $\omega_{\mathrm{TO}}<\omega_{\mathrm{SO}}<\omega_{\mathrm{LO}}$.

Samples were prepared over the glass matrix $\mathrm{SiO}_{2}$ $\mathrm{Na}_{2} \mathrm{CO}_{3}-\mathrm{B}_{2} \mathrm{O}_{3}-\mathrm{Al}_{2} \mathrm{O}_{3}$ doped with $\mathrm{CdO}, \mathrm{S}$ and Se. The mixture was melted in an aluminum crucible at $1200{ }^{\circ} \mathrm{C}$, for $2 \mathrm{~h}$. Then, it was quickly cooled down to room temperature. In or- der to enhance the diffusion of the $\mathrm{Cd}^{2+}, \mathrm{S}^{2-}$, and $\mathrm{Se}^{2-}$ ions, we have performed $600{ }^{\circ} \mathrm{C}$ thermal treatments on each samples using different annealing times of $t_{a}=3,5,6,10,12,14$, $16,18,20$, and $22 \mathrm{~h}$, respectively. As a result of these thermal treatments, the QD's of $\mathrm{CdS}_{x} \mathrm{Se}_{1-x}$ are formed over the glass matrix. The QD Raman spectra were recorded at room temperature. An argon-ion laser operating at $100 \mathrm{~mW}$ with the line $\lambda=514 \mathrm{~nm}$ was used for the Raman scattering. Finally, the scattered light was collected in the back scattering geometry, using a Jobin-Yvon triple spectrometer.

Owing to the small size in QD's, the surface-to-volume ratio contribution to the Raman spectrum is much higher than in bulk crystals. Therefore, in spheroidal nanocrystals surrounded by a glass matrix with a real frequency-independent dielectric constant $(\varepsilon(\omega))$, one might expect Raman observation of the SO-phonon modes with frequencies between the corresponding LO and TO bulk phonons frequencies. The main macroscopic quantities involved in the description of polar-optical phonons are derived from the equation $\varepsilon(\omega) \nabla^{2} \varphi=0$, where $\varphi$ is the electrostatic phonon potential. For ternary alloys, $\varepsilon(\omega)$ can be calculated as [2]

$$
\varepsilon(\omega)=\varepsilon_{\infty}+\frac{X_{1}}{\omega_{T 1}^{2}-\omega^{2}}+\frac{X_{2}}{\omega_{T 2}^{2}-\omega^{2}} .
$$

Here, the subscript 1 (2) refers to the CdSe-like (CdS-like) modes. The polarizability parameters for the two TO-modes can be written as

$$
\begin{aligned}
& X_{1}=(1-x) \varepsilon_{1 \infty}\left(\omega_{L 1, B}^{2}-\omega_{T 1, B}^{2}\right), \\
& X_{2}=x \varepsilon_{2 \infty}\left(\omega_{L 2, B}^{2}-\omega_{T 2, B}^{2}\right)
\end{aligned}
$$

where the subscript $B$ indicates that the phonon frequencies are those for binary compounds with $\varepsilon_{1 \infty}$ and $\varepsilon_{2 \infty}$. The LOphonon modes are obtained from $\varepsilon(\omega)=0$. Thus, Eq. (1) 
gives the LO frequencies of the CdS-like and CdSe-like modes as the roots of a quadratic function in $\omega_{L O(1,2)}^{2}$ Note that these frequency-modes are size-independent since the dielectric function only contains information of the constituent materials.

For the SO-phonons we need to solve the Laplace equation $\nabla^{2} \varphi=0$, where the solutions satisfy the usual electrostatic boundary conditions between two different media. The main results of the formalism developed for spheroidal QD's, in Ref. [1], can be summarized in the following equation

$$
\frac{\varepsilon(\omega)}{\varepsilon_{D}}=\left(\left.\frac{d}{d \xi} \ln Q_{l}^{m}\right|_{\xi_{0}}\right)\left(\left.\frac{d}{d \xi} \ln R_{l}^{m}\right|_{\xi_{0}}\right)^{-1}
$$

Note that this is the formal expression for the electrostatic boundary conditions of prolate spheroidal QD's with coordinates $\xi \geq 1,-1 \leq \eta \leq 1$, and $0 \leq \varphi \leq 2 \pi$. The condition $\xi=$ constant, defines an ellipsoid of revolution with the $z$-axis pointing along the ellipsoid's major axis. For $1 \leq \xi \leq \xi_{0}$, we have a $\mathrm{CdS}_{x} \mathrm{Se}_{1-x}$ QD with a dielectric constant, $\varepsilon(\omega)$, in the interior of the spheroid. The region $\xi \geq \xi_{0}$ defines the glass matrix with dielectric constant $\varepsilon_{D}$. The set of functions $Q_{l}^{m}$ and $R_{l}^{m}$, in Eq. (3), are related with the solutions of the Laplace equation for the $\xi \geq \xi_{0}$ and $\xi \leq \xi_{0}$ regions, respectively. The vibrational quantum numbers are given by $l=1,2,3, \ldots$ and $|m| \leq l$ (see Ref. [1] for further details). In addition, the limit $1 / \xi_{0} \rightarrow 0$ represents the purely spherical situation expressed as $\varepsilon(\omega)=-\varepsilon_{D}(l+1) / l$. Note that the right side of Eq. (3) depends on the QD geometrical shape through the spatial parameter $\xi_{0}$. The information about the nature of the constituent material is entirely contained in the dielectric functions.

We calculate the SO frequencies by solving Eq. (3) for a ternary compound dielectric function as defined in Eq. (1). In perfectly spherical QD's the SO frequencies are independent of the dot-size. However, for spheroidal QD's the SO-modes show appreciable variation with the size and the geometrical shape. In both cases, these modes depend strongly on the concentration $x$

In Fig. 1(a) we show the measured peak positions or Raman shift corresponding to the CdS-like and CdSe-like LO modes for different heating treatment times of the QD's. Experimental evidence confirm a clear tendency of increasing dot-size distribution when the heating time is increased (for a general review of growth processes see Ref. [3]). Fig. 1(b) shows the Raman profile for ternary $C d S S e$ QD with heating time of 16 $\mathrm{h}$. The presence of the expected SO-modes is clearly revealed by the asymmetry of Raman band with a tail towards lower energies and by the small red-shift of the peaks energies. On the other hand, the clear presence of surface phonons reveals, without ambiguity, the formation of QD's in the glass matrix, as these modes only appear as consequence of the strong spatial confinement.

We also show on Fig. 1(a) the difference $\Delta \omega$ between the $\mathrm{CdS}$ - and CdSe-like frequency modes. It is noted that these modes show an negligible dependence with the dot-size. The small frequency variation on $\Delta \omega$ corresponding to $\pm 2 \mathrm{~cm}^{-1}$ can be attributed to strain or small spatial confinement effects on the phonon modes [4]. The positions of the peaks corresponding to the SO-modes, as a function of the heating times,
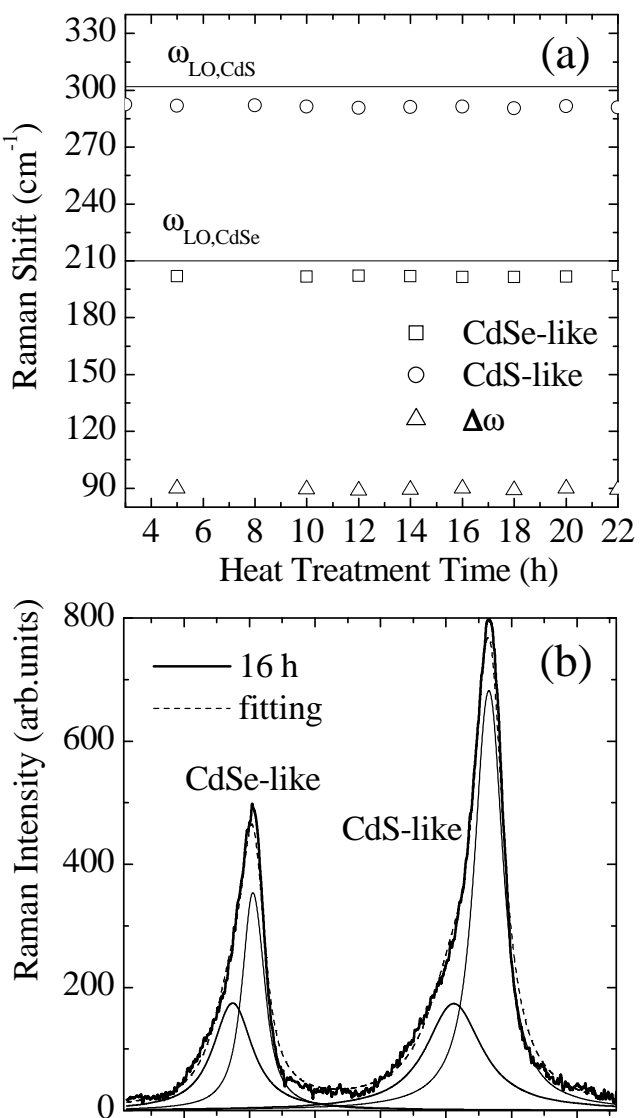

175200225250275300325

Raman Shift $\left(\mathrm{cm}^{-1}\right)$

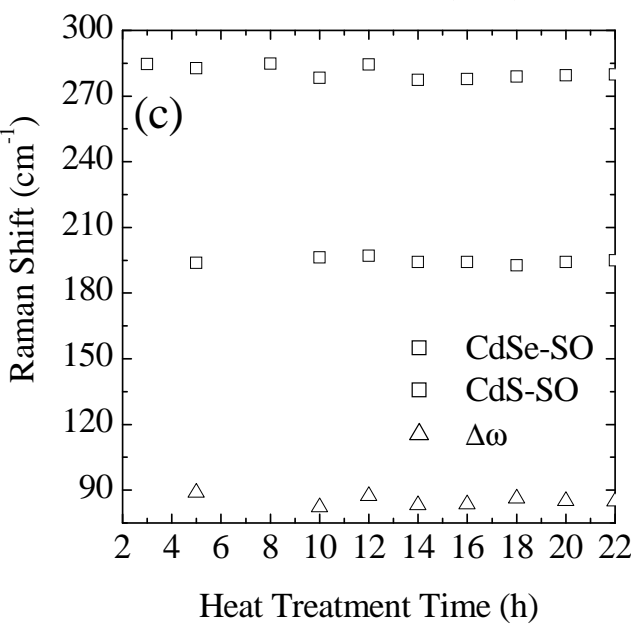

FIG. 1: (a) Experimental Raman shift for CdS- and CdSe-like LOmodes after different heating times. Solid lines show LO and TO bulk frequencies. (b) Raman profile for a ternary CdSSe QD with annealing time $t_{a}=16 \mathrm{~h}$. (c) Same as in (a) for CdS and CdSelike SO-modes. The triangles, in (a) and (c), show $\Delta \omega=\omega_{L O, S O}^{\mathrm{CdS}}-$ $\omega_{L O, S O}^{\mathrm{CdSe}}$. Note also the fittings for LO- and SO-modes. 


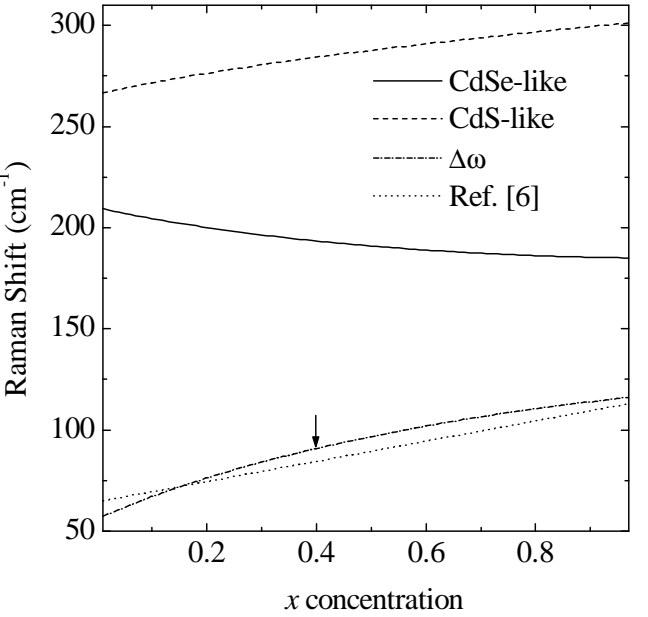

FIG. 2: CdS- and CdSe-like phonon modes calculated from $\varepsilon(\omega)=0$. The frequency difference $\Delta \omega$ is displayed in dashed-dotted lines. The dotted lines represent the phenomenological relation, $x=0.020 \Delta \omega$ 1.29, taken from Ref. [6]. The arrow indicates the approximate value of the concentration $x$.

are presented in Fig. 1(c). The set of data points labeled as $\Delta \omega$ represents the difference between the SO-CdS and SO$\mathrm{CdSe}$ modes. It is noted that the SO modes are sensitive to dot-size change. Considering that purely spherical QD's do not have size dependent phonon frequencies, we can conclude that the shape of the nanocrystals are deviating from spherical to ellipsoidal-like.

Next, we compare the experimental data with our theoretical results in order to determine the mean value of the concentration $x$ and the degree of deviation from a pure spherical shape. To estimate the LO and SO frequencies of the ternary $\mathrm{CdS}_{x} \mathrm{Se}_{1-x}$ nanocrystals we analyze the frequency TO-modes of CdSe-like and CdS-like phonons, that vary nearly linearly with concentration $x$, from $\omega_{T O, 1}=168+17 x \mathrm{~cm}^{-1}$, and $\omega_{T O, 2}=266-28 x \mathrm{~cm}^{-1}$. The LO and TO frequencies for binary CdSe and $\mathrm{CdS}$ compounds are $\omega_{L O, 1}=210 \mathrm{~cm}^{-1}$, $\omega_{L O, 2}=302 \mathrm{~cm}^{-1}, \omega_{T O, 1}=168 \mathrm{~cm}^{-1}$, and $\omega_{T O, 2}=238$ $\mathrm{cm}^{-1}$ [5]. The dielectric constants are $\varepsilon_{\infty, 1}=1.61, \varepsilon_{\infty, 2}=5.32$ while for the host material, we are taking $\varepsilon_{D}=2.25$ [4]. Figure 2 shows the calculated frequencies for CdSe-like and CdSlike TO modes as a functions of the concentration $x$. The line labeled $\Delta \omega$ represents the difference between these modes. A simple quadratic fit shows that the relation between $\Delta \omega$ and $x$, is given by $\Delta \omega=57.64+98.6 x-40.31 x^{2}$. For comparison purposes we are showing, in dotted line, the best fit obtained by Miyoshi et al. [6] from their analysis of Raman scattering data for several concentrations $x$. By comparing the experimental data presented in Fig. 1(b) with the theoretical results of Fig. 2 we obtain the difference $\Delta \omega$, as approximately $89.0 \pm 2.0 \mathrm{~cm}^{-1}$. This value corresponds to a concentration $x=0.39 \pm 0.02$. Moreover, a large systematic error exists when using only one single mode to determine the composition, since strain and phonon confinement effects displaces the CdS-like and CdSe-like modes in the same direction. In

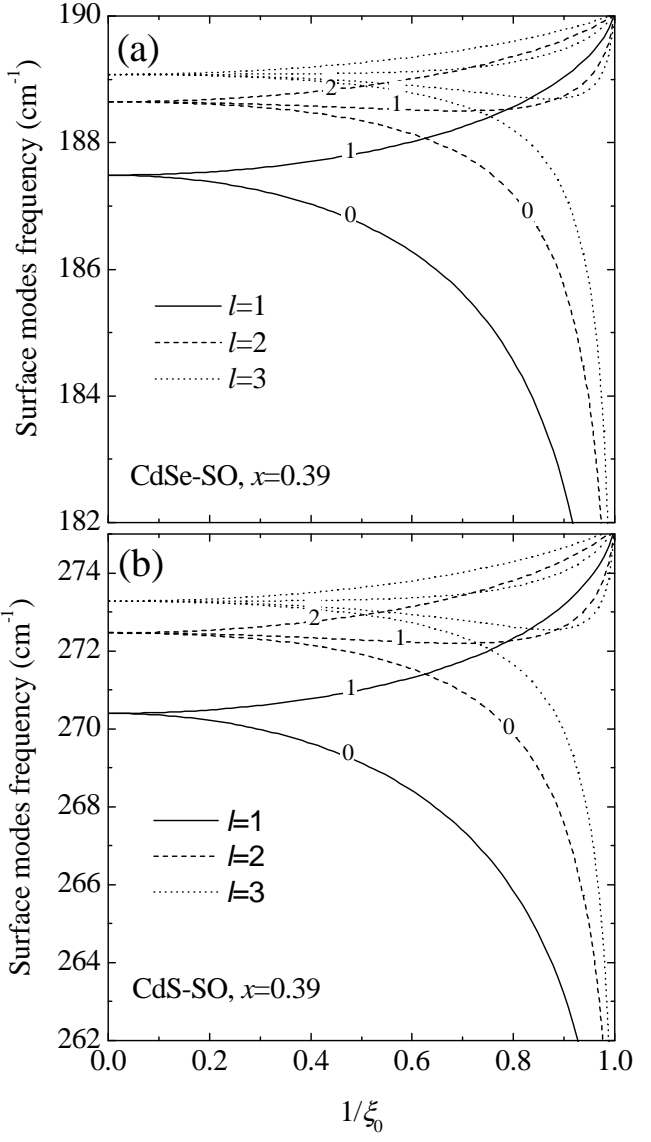

FIG. 3: (a) CdSe-SO frequencies as a function of $\xi_{0}^{-1}$ for $l=1,2,3$ for all possible values of quantum number $m$ of a prolate ellipsoidal QD. (b) Same for CdS-SO modes. The calculations were performed with $x=0.39$.

contrast, the compositional change causes them to shift in opposite directions. In order to reduce the systematic error we are using the difference between the two modes (CdS-like and CdSe-like) to determined the concentration $x$. These arguments can also be applied to the SO-mode case to determine the geometric characteristics of the QD's.

The dependence with the spatial parameter $\xi_{0}$ for the CdS and CdSe SO frequencies of QD's,calculated for $x=0.39$, is shown on Figs. 3(a) and 3(b). We consider the modes with $l=1,2,3$. The numbers over the lines indicate the corresponding quantum number $m$. The limit $\xi_{0}^{-1} \rightarrow 0$ corresponds to the pure spherical case. Note the splitting of the SO frequencies according to the condition $|m| \leq l$ as well as the strong dependence with the dimensions of the dot through the spatial parameter $\xi_{0}$. It is interesting to recall that spherical modes are $2 l+1$-degenerated and do not exhibit spatial sensitivity. Another important aspect is to determine which is the predominant phonon mode in the Raman response. For non-spherical QD's, the Raman selection rules only allow SOphonons with $m=0$ and $l=$ even integer $[1,7]$. The mode $l=1$ is not allowed. However, defects or impurities in the QD may relax this rule and induces Raman scattering with the par- 


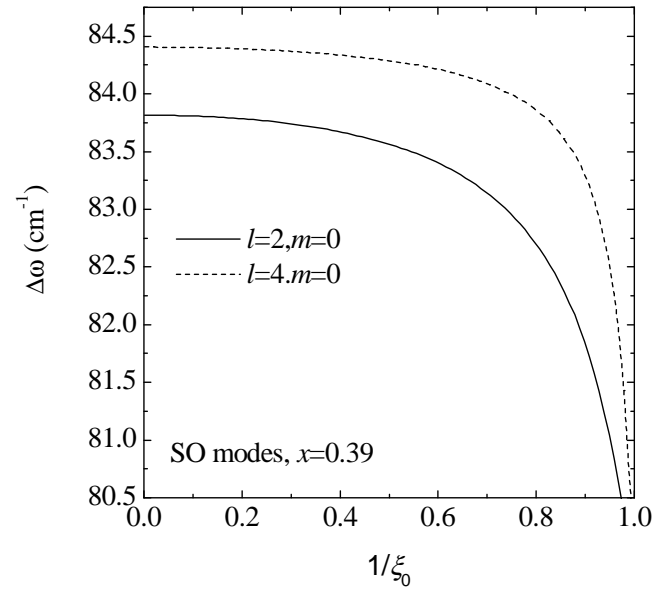

FIG. 4: Frequency difference for $\mathrm{CdS}$ and $\mathrm{CdSe} \mathrm{SO}$ as a function of the spatial parameter $\xi_{0}^{-1}$, for $l=2, m=0$ - and $l=4, m=0$-modes.

ticipation of $l=1$-modes. In order to analyze the geometric properties of the QD, we will restrict our attention only to the selection rule $l=$ even, $m=0$. Fig. 4 shows the dependence between the difference of the CdS and CdSe SO frequencies with the spatial parameter $\xi_{0}$, for the $l=2, m=0$ and for $l=4, m=0$ cases.

Let us compare our theoretical results for the samples in Fig. 1(c), with heating times: $16 \mathrm{~h}\left(\Delta \omega_{S O}=83.56 \mathrm{~cm}^{-1}\right)$, $14 \mathrm{~h}\left(\Delta \omega_{S O}=83.20 \mathrm{~cm}^{-1}\right)$ and $10 \mathrm{~h}\left(\Delta \omega_{S O}=82.14 \mathrm{~cm}^{-1}\right)$. The corresponding parameters $\xi_{0}$ and ellipsoid's semiaxis ratio $r=\xi_{0}\left(\xi_{0}^{2}-1\right)^{-1 / 2}$ for the $l=2, m=0$ SO-modes are: (i) for the sample with $16 \mathrm{~h}$ of heating treatment, we obtained $\xi_{0}=2.04$ and $r=1.14$; (ii) for the one with $t_{a}=14 \mathrm{~h}$ we have $\xi_{0}=1.49$ and $r=1.34$; (iii) for $10 \mathrm{~h}$ of heat treatment we have gotten $\xi_{0}=1.14$ and $r=2.08$. Important points can be derived from these results: a) for the $l=2 \mathrm{SO}$-modes, when the deviation of the spherical shape increases the difference between the SO frequencies $\left(\Delta \omega_{S O}\right)$ diminishes. b) These QD's present strong deviations from the spherical shape. For instance, for $l=2, m=0$ we obtain semi-axis ratio in the interval: $1.14 \leq r \leq 2.18$. c) The large size QD's (large heating time treatments) present shapes closer to spherical geometry, once $r$ is decreasing with increasing heating time. The samples with $\left(\Delta \omega_{S O}=84.50 \mathrm{~cm}^{-1}\right)$ have not been considered; this large energy difference would suggest that SO modes $l=1$ are excited in the Raman process. Although these modes are forbidden for the Raman selection rules in non-spherical shapes, they can be excited by the presence of impurities or defects created during the nucleation process. In general, modes with $l>2$ present small contribution to the Raman response and have not been thoroughly considered in the present analysis.

In conclusion, we have performed Raman spectra measurements where the presence of surface modes associated to CdSlike and CdSe-like phonon branches is observed. The frequencies of these modes are dependent on the concentration $x$ and on the time of thermal treatment; these results suggest that the quantum dots are not perfectly spherical in shape. We compared our theoretical results with experimental Raman spectra and determined the molar concentration of our samples to be $x=0.39$. In addition, the shape of the QD's exhibits a very strong deviation from the spherical geometry with a range of $1.14 \leq r \leq 2.18$ for the selected set of samples.

Authors acknowledge financial support from the agencies: Conselho Nacional de Desenvolvimento Cientfico e Tecnolgico $(\mathrm{CNPq})$ and Fundação de Amparo à Pesquisa do Estado de Minas Gerais (FAPEMIG).
[1] F. Comas, C. Trallero-Giner, N. Studart, and G. E. Marques, Phys. Rev. B 65073303 (2002).

[2] K. J. Nash, M. S. Skolnick, and S. J. Bass, Semicond. Sci. Technol. 2329 (1987)

[3] U. Woggon,Optical Properties of Semiconductor Quantum Dots, Springer, Berlin, 1997.

[4] A. V. Gamonnai, Yu. M. Azhniuk, V. O. Yukhymchuck, M. Kran- jcec, and V. V. Lopushansky, Phys Stat. Sol (b) 239490 (2003).

[5] A. Ingale and K. C. Rustagi, Phys. Rev. B 587197 (1998).

[6] T. Miyoshi, T. Nakatsuka, and N. Matsuo, Japn. J. Appl. Phys. 341835 (1995).

[7] F. Comas, C. Trallero-Giner, N. Studart, and G. E. Marques, J. Phys: Condens. Matter 146469 (2002). 